\title{
Espejismo en el desierto: las fallas en la primera fase de la intervención estadounidense en Irak*
}

\author{
Mirage in the desert: the flaws in the first phase of U.S. involvement \\ in Iraq \\ Recibido: 20 de septiembre de 2011 - Revisado: 06 de octubre de 2011 - Aceptado: 29 de octubre de 2011
}

Fabio Sánchez Cabarcas**

\begin{abstract}
Resumen
La intervención estadounidense en Irak desde 2003 constituye uno de sus últimos desafíos en su política exterior. Bajo un marcado unilateralismo y sin el aval de la ONU, la Administración de G. W. Bush decidió invadir a un actor fundamental en el equilibrio de poder de Oriente Medio. Su lucha contra el terrorismo internacional ocasionó resultados adversos en Irak: crecimiento de la insurgencia y el fundamentalismo, fallas administrativas en la reconstrucción (ORHA y APC) y acciones militares en contra de la población civil en un enfrentamiento irregular. Estos factores evidencian una política errónea de Washington hacia ese país y la región.
\end{abstract}

Palabras clave

EE. UU., G. W. Bush, ORHA, APC, insurgencia.

\begin{abstract}
The U.S. intervention in Iraq since 2003 is one of their last challenges in its foreign policy. Under a strong unilateralism and without UN approval G.W. Bush Administration decided to invade a key player in the balance of power in the Middle East. In its fight against international terrorism, the outcomes are adverse: growth of the insurgency and fundamentalism, administrative failures in the reconstruction (ORHA and CPA), and military actions against civilians in an irregular conflict. These are factors that account of a failed American policy. Key words

U.S., G.W. Bush, ORHA, CPA, insurgency.
\end{abstract}

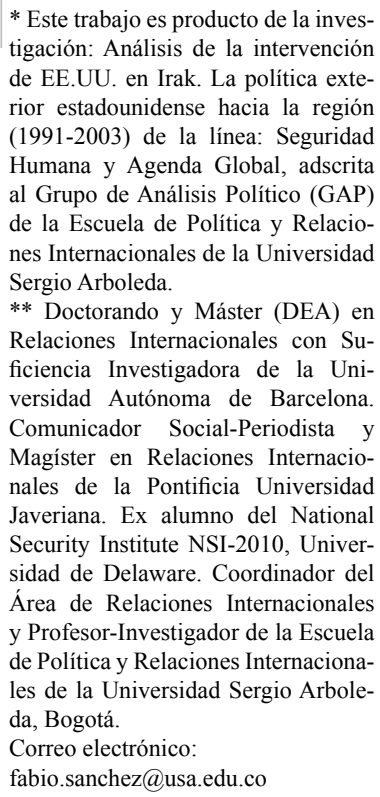

* Este trabajo es producto de la investigación: Análisis de la intervención de EE.UU. en Irak. La política exterior estadounidense hacia la región (1991-2003) de la línea: Seguridad Humana y Agenda Global, adscrita al Grupo de Análisis Político (GAP) de la Escuela de Política y Relaciones Internacionales de la Universidad Sergio Arboleda.

** Doctorando y Máster (DEA) en Relaciones Internacionales con $\mathrm{Su}-$ ficiencia Investigadora de la Universidad Autónoma de Barcelona. Comunicador Social-Periodista y Magíster en Relaciones Internacionales de la Pontificia Universidad Javeriana. Ex alumno del National Security Institute NSI-2010, Universidad de Delaware. Coordinador del Área de Relaciones Internacionales y Profesor-Investigador de la Escuela de Política y Relaciones Internacionales de la Universidad Sergio Arboleda, Bogotá.

Correo electrónico:

fabio.sanchez@usa.edu.co 


\section{Introducción}

En la primera fase de su Administración G. W. Bush enfocó la política exterior hacia temas regionales. De ahí, su iniciativa al diálogo con vecinos próximos. El 16 de febrero de 2001 hizo su primer viaje al extranjero, reuniéndose con su homólogo mexicano Vicente Fox. También fue evidente la promoción de ciertos objetivos realistas relativos al interés nacional: la instalación de un Escudo anti-Misiles, el fortalecimiento del ejército y el refuerzo de alianzas con Europa, Japón y Taiwán, con el propósito de contener a China. Estas prioridades pasaron a un segundo plano cuando se inició la lucha mundial contra el terrorismo, luego del 11-S (Kupchan, 2003, p. 233).

El 11-S significó un ataque crítico a la seguridad de EE. UU. a través de la tecnología y redes de información interdependientes. Al Qaeda propició un golpe sofisticado sin precedentes en la historia moderna que evidenció una asimetría informativa negativa por parte de Washington hacia el resto del mundo (Keohane, 2002). Esto ocasionó un giro violento en la actitud del gobierno y la opinión pública estadounidense, tanto republicanos como demócratas se aliaron en la lucha contra el terrorismo internacional, apoyando una política exterior unilateralista e intervencionista.

El 14 de septiembre de 2001, G. W. Bush se dirigió al Congreso en tono enérgico y manifestó: "Únanse a nosotros en nuestra cruzada o confronten la perspectiva segura de muerte y destrucción; quien no esté con nosotros, estará contra nosotros" (Apple, 2001). Días después, el 20 de septiembre, elaboró un discurso aún más agresivo dirigido a la comunidad internacional, en el que manifestó: "Están con nosotros o con los terroristas. Están con la civilización y los buenos (nosotros) o con los bárbaros y el mal (ellos). Elijan. Y aquellas naciones que elijan mal, tengan cuidado" (Hirsh, 2002, p. 19).

La respuesta de EE. UU. fue rápida, sus servicios de inteligencia junto con los británicos empezaron a relacionar el 11-S con un grupo terrorista desconocido para muchos hasta el momento: Al Qaeda ${ }^{1}$. Su jefe, un millonario saudí, Osama Bin Laden, quien durante la década de 1980 recibió apoyo de Washington para expeler a las tropas soviéticas de Afganistán, en donde, curiosamente, inició la respuesta militar de EE. UU. en $2001^{2}$.

Los servicios de inteligencia de EE. UU. se basaron en indicios para asegurar que el centro de operaciones de Bin Laden y su grupo estaba en Afganistán. En teoría recibían apoyo y protección del Régimen Talibán, el cual se caracterizaba por su opresión contra las mujeres, la constante violación de los Derechos Humanos y una forma de gobierno déspota que hacía una interpretación extrema del Corán. A estos graves antecedentes, se suma el presumible apoyo que el gobierno de Pakistán brindaba a la organización de Bin Laden, y otros hechos abominables como la destrucción de las milenarias estatuas de los Budas Gigantes de Bamiyán, haciendo caso omiso a múltiples llamados de atención de la UNESCO ${ }^{3}$.

Luego de una fuerte protesta internacional en contra de la guerra, el 7 de octubre de 2001 inició la Operación Libertad Duradera. Contó inicialmente con un presupuesto aproximado de 40.000 millones de dólares y el apoyo de 40 países aliados. Su objetivo fue bombardear escondites de Al Qaeda en las montañas de Afganistán, capturar a Bin Laden y derrocar al Régimen Talibán.

Esta operación fue la primera fase de la lucha contra el terrorismo global. Por su parte, el entonces Primer Ministro británico Tony Blair manifestó que no se trataba de una lucha contra el Islam y que se evitaría a toda costa la muerte de civiles. Si bien las condiciones geográficas y climáticas dificultaron el avance militar (tormentas de arena y complejos sistemas montañosos), las tropas estadounidenses y sus aliados, con el apoyo de la Alianza Norte, llegaron a Kabul el martes 13 de noviembre de 2001 
a las 14:51 (GMT). Los bombardeos aéreos de forma simultánea ya habían debilitado la infraestructura y redes de comunicación de los talibanes (Sohr, 2002, p. 9). Se trató en principio de una operación quirúrgica con una posterior operación terrestre larga y compleja.

Los prisioneros detenidos pertenecientes a Al Qaeda fueron llevados a la prisión de Guantánamo en Cuba. Acto que fue duramente criticado por la opinión pública internacional, manifestando que se trataba de una violación a los Derechos Humanos. El "relativo" éxito militar no produjo ni la captura de Bin Laden ni la del Mulá Mohammed Omar, máximo líder talibán en Afganistán; una muestra de las dificultades propias que implica la guerra irregular de guerrillas que se desarrolló en Irak desde 2003.

Una vez debilitado el Régimen Talibán, Washington instaló como presidente interino a Hamid Karzai. Un excéntrico miembro de la exiliada realeza afgana que tuvo la misión de consolidar un sistema de gobierno democrático a través de planes económicos que buscaban sacar al país de la crisis económica para convertirlo en una pieza clave en la geoestrategia de Washington ${ }^{4}$.

Si bien esta campaña militar fue rápida y mostró a EE. UU. como un actor efectivo, las limitaciones fueron muy grandes. Principalmente se evidenció que el terrorismo era un enemigo complejo de combatir y derrotar: los líderes de Al Qaeda no fueron capturados. Y algunos medios de comunicación presentaron informes que alarmaron a los dirigentes en Washington: la posibilidad de que Bin Laden y su grupo estuvieran refugiados en Pakistán, país que hábilmente se habría aliado con EE. UU. para hacerse intocable y protegerlos durante y después de la guerra (Fuentes, 2003, p. 12). Otro caso más de hipocresía contra EE. UU. en el Mundo Árabe, como ha ocurrido en Egipto y Arabia Saudita, en donde el wahabbismo ha convertido al antiamericanismo ${ }^{5}$ en un chivo expiatorio que oculta su propia corrupción.
El 29 de enero de 2002, durante el discurso de la Unión, G. W. Bush acuñó la expresión "eje del mal", haciendo referencia a regímenes que daban apoyo al terrorismo: Irak, Irán y Corea del Norte ${ }^{6}$. No obstante, la vulnerabilidad de EE. UU. continuó siendo evidente, varios ataques con ántrax en oficinas postales generaron un pánico general en la población, lo que para Gause representa el hecho que más influyó en la decisión de atacar a Irak (Gause, 2010, p. 191)7.

En febrero de 2002, un mes después, se ordenó a la CIA elaborar un plan para sacar a Hussein, incluso a través de la fuerza. En Washington pensaban que existían pocas posibilidades de que Irak diera Armas de Destrucción Masiva (WMD, por sus siglas en inglés) a Al Qaeda, pero de ser posible, así fuera el $1 \%$, las consecuencias serían fatales lo cual justificó la guerra anticipatoria y la "Doctrina del 1\%". Otros miembros del staff neocon ${ }^{8}$ en Washington pensaron que un Oriente Medio sin Hussein favorecería a EE. UU., que su derrocamiento enviaría un mensaje claro a otros enemigos y que luego iniciaría una fase de democratización en la región (Gause, 2010, pp. 238-239). Nada más alejado de la realidad, tal y como se explicará a continuación.

Varios factores dan cuenta del fracaso de la intervención estadounidense en Irak durante la primera fase de la Administración G. W. Bush. Los daños al equilibrio de poder regional establecido durante la Guerra Fría, el crecimiento de la insurgencia y la influencia del fundamentalismo islámico patrocinado por Irán, dan cuenta de las amenazas para Washington y sus aliados en la región. El artículo continúa de la siguiente manera: en la primera parte se analiza el papel de la Oficina para la Reconstrucción y Asistencia Humanitaria (ORHA, por sus siglas en inglés). Posteriormente, se examinan las acciones de la Autoridad Provisional de la Coalición (APC). En la tercera parte, se estudia la lucha contra la insurgencia, especialmente contra la fuerza de Moqtada al-Sadr y los fuertes enfrentamientos que se presentaron en las 
ciudades de Faluya y Nayaf en 2004. Finalmente, las conclusiones.

\section{La Oficina para la Reconstrucción y Asistencia Humanitaria (ORHA, por sus siglas en inglés) y la primera fase de la intervención}

El 20 de marzo de 2003 inició la Operación Libertad Iraquí. Con tres objetivos principales: i) derrocar a Hussein, ii) tomar el control del petróleo iraquí ${ }^{9}$ y iii) mejorar la seguridad de la región. Esto obligó a Washington a desarrollar una estrategia defensiva y militar, a través del Ejército y organismos de seguridad, para enfrentar las amenazas propias del terrorismo transnacional.

Los nuevos lineamientos en defensa habían sido expuestos por el Secretario de Defensa de aquel entonces Donald Rumsfeld en un artículo publicado en Foreign Affairs en 2002 titulado: "Transforming the Military". Los seis objetivos contemplados eran:

(...) i) proteger el territorio de EE. UU. y las bases continentales, ii) proteger y mantener el poder en zonas lejanas, iii) negar a los enemigos la capacidad de esconderse, sea en las montañas, búnkeres o cualquier otro lugar, nunca permitir que estén fuera de alcance, iv) proteger las redes de información, v) usar la tecnología de información para unir las distintas clases de fuerzas de EE. UU. para su lucha en grupo, y vi) no permitir el acceso al espacio y proteger las bases espaciales de ataques enemigos (Rumsfeld, 2002, p. 26).

Rumsfeld también destacó que esta estrategia no solo implicaba ganar guerras, sino también prevenirlas. Y frente al terrorismo, señaló que la mejor defensa consiste en una gran ofensiva, es decir, ataques anticipatorios. Asimismo, la Administración de G. W. Bush reorganizó el Departamento de Defensa y el Pentágono. Adoptó un esquema basado en el equilibrio de riesgos, a través del desarrollo de nuevos esquemas de protección y disuasión con los que se buscó incrementar la seguridad del país. El presidente G. W. Bush en su discurso del Estado de la Unión influenció a la opinión pública a través de la moral, religión y valores (visto desde el constructivismo) con los que afectó la toma de decisión de la opinión pública local: "La libertad no es un regalo de América al mundo, es un regalo de Dios a la humanidad (...) no conocemos los caminos a la Providencia, sin embargo, podemos confiar en ellos y en el amor de Dios, quien está detrás de la vida y la historia" (Dunn, 2003, p. 291 $)^{10}$.

Bajo órdenes directas de G. W. Bush, el Subsecretario de Defensa de Asuntos Políticos, Douglas Feith, planificó la invasión de Irak. También dirigió una oficina secreta denominada "Oficina de Planes Especiales", en la que se analizaron todos los informes de los servicios de inteligencia (Chandrasekaran, 2006a, p. 44). Una de sus tareas fue conseguir información que conectara a Hussein con Al Qaeda. El trabajo casi secreto de Feith evidenció la poca coordinación entre el Departamento de Estado, la CIA y el Pentágono. Esto tuvo, más adelante, graves consecuencias en los nombramientos de personas y su fracaso en la posguerra.

Uno de los casos más críticos al respecto fue el de Ahmed Chalabi ${ }^{11}$. Él dirigía desde Londres el Congreso Nacional Iraquí (CNI), un grupo disidente que tenía la simpatía del Departamento de Estado y la CIA. Al respecto, Wills manifiesta:

Creando nuevos procedimientos de inteligencia, la oficina del Vicepresidente Cheney y el Departamento de Defensa de Donald Rumsfeld hilaron finamente información a la CIA y la NSA canalizando inteligencia en bruto de fuentes como Ahmed Chalabi y Richard Perle. 
Esto originó la falsa información sobre laboratorios de armas móviles y centrifugadoras, presentada con tal miedo, que la Administración pidió un alto a las inspecciones de Hans Blix y la ONU sobre WMD (Wills, 2010, p. 224).

No obstante, tanto Chalabi como el CNI tenían una imagen negativa en la mayoría de los iraquíes, quienes lo veían como un corrupto y oportunista con deudas pendientes en Jordania por un descalabro económico años atrás. Hábilmente, Chalabi vendió la idea de un discurso que les gustó a los círculos neoconservadores en Washington: la creación y consolidación de una democracia secular en Irak, aliada con Occidente y que reconocería a Israel. Días antes de la ocupación, el propio Chalabi arengó un peligroso y profético discurso en Nasiriya: "No queremos vengarnos de nadie, pero debemos erradicar para siempre el partido Baas de Hussein ${ }^{12}$; como se verá más adelante, este será uno de los peores errores cometidos por las fuerzas extranjeras.

Feith, al igual que muchos en Washington y Occidente, pensaron que solo se trataba de una guerra de liberación, que implicaría una corta transición con una ayuda leve hasta que los propios iraquíes asumieran el control. Para ello, Feith llamó el 17 de enero de 2003 a Jay Garner, un Teniente General retirado, para que se hiciera cargo de Irak en la posguerra, la cual se calculaba duraría tan solo 90 días (Chandrasekaran, 2006a, p. 45). Garner tenía experiencia previa en Irak. Durante la Guerra del Golfo en 1991 comandó una operación para proteger a los kurdos en el norte, no obstante, cuando llegó al Pentágono no tenía ni personal ni presupuesto y le fue asignado, según sus propias palabras, "el cuarto de escobas” (Chandrasekaran, 2006a, p. 45).

La principal tarea de Garner fue dirigir la ORHA. La dividió en tres pilares: i) ayuda humanitaria, ii) reconstrucción y iii) administración civil. Determinó tres zonas administrati- vas, las cuales no tenían relación con los límites de las provincias iraquíes ${ }^{13}$ ni con la ubicación de las fuerzas militares. En principio tenían planeado salir en menos de 90 días, facilitando una rápida transición, removiendo al régimen pero dejando al Estado funcionando (Gause, 2010, pp. 155-156).

El equipo tenía una compleja composición: reservistas, personal de la Agencia Estadounidense para el Desarrollo Internacional (USAID, por sus siglas en inglés), funcionarios gubernamentales civiles y personal del Departamento de Estado, desde donde insistían en trasladar a algunos de sus diplomáticos. En el equipo algunos eran de gran calidad y otros, excedentes de la burocracia federa ${ }^{14}$, todos con buenas intenciones pero con poca experiencia en Oriente Medio, y lo que es peor, en sus áreas de trabajo (Chandrasekaran, 2006a, pp. 46-48).

Garner nunca recibió algún tipo de plan elaborado por Feith. Tampoco documentos para la posguerra elaborados por el Departamento de Estado, ni análisis de la CIA, o informes confidenciales de militares de la Universidad de la Defensa Nacional, en donde se adelantó un seminario de dos días con 70 académicos y especialistas.

El Departamento de Estado había desarrollado un plan para Irak, por medio de la Oficina de Asuntos del Cercano Oriente. Se trató de un ejercicio de planeación política con 17 temáticas trabajadas con más de 200 exiliados iraquíes (Le Billon, 2005, p. 695). Se abordaron temas a través de grupos de trabajo enfocados en temas como medidas anticorrupción y energía y petróleo. Pero en enero de 2003, después de que G. W. Bush firmó una Directiva de Política de Seguridad Nacional, el tema fue centralizado e ignorado por el Pentágono (Dodge, 2005, p. 711; Le Billon, 2005, p. 695).

Garner le insistió a Feith sobre los documentos, pero este le dijo que no eran importantes y que debería elaborar sus propios planes. 
La estrategia de Feith consistía en que Garner, sin un plan claro, acudiera a Chalabi y su grupo de exiliados. De esta manera lograría su objetivo, sin generar choques entre el Departamento de Estado y la CIA, en donde se consideraba a Chalabi como un farsante (Chandrasekaran, 2006a, p. 2). Al respecto, Dodge afirma:

Chalabi fue un promotor clave de la tesis de decapitación puesta en el corazón de los planes de la posguerra en Irak (...) argumentó que el gobierno de Hussein era altamente vulnerable e inestable, que no tenía raíces ideológicas ni institucionales dentro de la sociedad iraquí, que dependía de poca gente para mantenerse en el poder (Dodge, 2005, p. 711).

Este falso análisis de Chalabi hizo creer en las esferas de Washington que un rápido ataque aéreo sacaría a Hussein del poder. Y además, que los iraquíes recibirían con los brazos abiertos a la fuerza estadounidense (al principio fue así). Bajo esta aproximación no eran necesarios más efectivos, pero la insurgencia y guerra de guerrillas que se desataron más adelante hicieron que la tropa en suelo iraquí se viera diezmada y en ocasiones superada por la resistencia local.

No obstante, Garner trabajó como poseso en su plan a finales de 2002 y principios de 2003. Debido a su experiencia tenía claro que la primera fase de la "corta" guerra generaría enfermedades, desplazamientos y hambrunas en miles de iraquíes, por tanto, su esfuerzo se centró en la consolidación de una estrategia humanitaria (Chandrasekaran, 2006a, p. 47). En ese momento, el Subsecretario de Defensa Paul Wolfowitz nombró a Timothy Carney, embajador retirado, en la ORHA, quien de inmediato se ofreció como Defensor del Pueblo, pero los planes del Departamento de Estado era nombrarlo provisionalmente como Ministro de Industria y Minerales, sin tener experiencia al- guna en ese sector, algo que poco importaba en Washington. Al respecto Dodge:

El falso supuesto de que las fuerzas de EE. UU. podrían utilizar, una vez en Bagdad, las instituciones iraquíes en funcionamiento, fue uno de los errores de cálculo que socavaron el enfoque de la coalición para la construcción del Estado. La combinación de una comprensión de la posguerra de Irak con carga ideológica y la falta de preparación e investigación sobre el país antes de la invasión, dio lugar a sus consecuencias catastróficas (Dodge, 2005, p. 710).

Las labores de reconstrucción fueron asignadas a un equipo de USAID. La administración civil, que resultó ser la labor más importante, fue delegada a Michael Mobbs, un viejo socio del buffet de abogados de Feith. El Ministerio de Comercio se asignó a otro embajador sin previa experiencia en el área y el Ministerio de Educación, a un burócrata de nivel medio del Departamento del Tesoro.

Otros hechos evidencian la confusión en el montaje de la ORHA. Stephen Browning, miembro del Cuerpo de Ingenieros del Ejército, quedó encargado de los ministerios de: Transporte, Comunicaciones, Vivienda, Construcción, Irrigación y Electricidad, y por ejemplo, el asistente de Carney tenía bajo su mando a casi mil empleados.

¿Cómo se concebía a la ORHA desde Washington? El Secretario de Estado Collin Powell y el Subsecretario Richard L. Armitage querían en esta oficina a diplomáticos expertos que hablaran árabe y entendieran las dinámicas propias del país y la región. Para el Departamento de Estado era importante contar con los iraquíes que habían permanecido en el país durante el régimen de Hussein, ya que tenían el conocimiento del funcionamiento de las instituciones gubernamentales y de la sociedad misma. No obstante, en el Pentágono consideraban 
que los arabistas de la vieja escuela pertenecientes al Departamento de Estado solo buscaban excusas para justificar que la democracia no funcionaría en Oriente Medio y que Chalabi y sus colegas dirigirían una democracia estable y secular (Chandrasekaran, 2006a, p. 49).

El 17 de marzo de 2003, dos días antes de que Garner partiera hacia Kuwait, Rumsfeld analizó el equipo que él y Feith y habían seleccionado. Según Chandrasekaran, Rumsfeld llamó a Garner y le dijo: "No me siento cómodo con estos tipos", a lo que éste replicó: "Es un poco tarde, salimos mañana". No obstante, Rumsfeld se comprometió a buscar nuevos miembros y canceló la salida de personal de alto rango del Departamento de Estado que había sido asignado a la ORHA" (Chandrasekaran, 2006a, p. 49).

Garner y su "equipo editado" llegaron a Kuwait el 19 de marzo de 2003. Es decir, 24 horas antes de iniciar la guerra. Para él era primordial distribuir agua y comida con el propósito de paliar el drama humanitario, propio de los conflictos en su primera fase ${ }^{15}$. Pero en Washington el plan era diferente. El 20 de marzo a las 5:30 a.m. sonaron las alarmas antiaéreas en Bagdad, reaccionado al paso de un misil crucero. Luego de 45 minutos, 6:15 a.m. (10:15 p.m. en EE. UU.), el Presidente G. W. Bush declaró el inicio de los bombardeos. En ese momento, y con justa razón, algunos expertos vaticinaron que la guerra urbana podría ser el "talón de Aquiles" de EE. UU.

El 21 de marzo Bagdad ardía en llamas. Fueron destruidos los ministerios de Información y Planeación (en donde, en teoría, deberían trabajar los miembros de las ORHA). Se calcula que el siguiente día cayeron 1500 bombas sobre diversos puntos del país. Uno de los palacios presidenciales fue incendiado, mientras que las tropas aliadas se tomaron el puerto estratégico de Um Qasar, único con salida al mar.

El 23 de marzo fue sitiada Basora, la capital petrolera del país. Se estima que cayeron 300 misiles sobre puntos estratégicos en Bagdad. Asimismo, 15 pozos fueron incendiados como táctica de guerra por los propios iraquíes. El 24 continuaron fuertes bombardeos y cayeron los primeros soldados estadounidenses muertos. En ese momento la comunidad internacional se preguntó: ¿en dónde están las WMD de Hussein? Ningún miembro de la Administración Bush tuvo la respuesta.

Las tropas estadounidenses encontraron resistencia camino a Bagdad. Los enfrentamientos en Basora generaron la huída de 13.000 personas. El 30 de marzo, el General Tommy Franks manifestó que se encontraban a 80 kilómetros de Bagdad. El primero de abril se presentaron fuertes combates en Hilla, a 80 kilómetros al sur de Bagdad, y el 2 de abril aviones B-52 lanzaron seis bombas de racimo, de 450 kilogramos cada una, sobre una columna de tanques que defendía la ciudad. Asimismo, la tercera división de infantería derrotó a la División Bagdad de la Guardia Republicana en Karbala.

El 3 de abril, Bagdad perdió la electricidad tras fuertes explosiones en el centro y sur de la ciudad. Las fuerzas de EE. UU. tomaron el control del Aeropuerto Internacional Saddam, situado a 20 kilómetros al suroeste de la capital, y tomaron el control de un puente estratégico sobre el Éufrates que comunicaba con Karbala. También se allanó el palacio presidencial de Tharthar, una de las residencias favoritas de Hussein.

El 5 de abril las fuerzas entraron en Bagdad. Aproximadamente 50.000 hombres apoyados con tanques y helicópteros de asalto chocaron con el ejército iraquí en el barrio Dora. Los británicos ingresaron a Basora y dos días después tenían el $80 \%$ del control de la ciudad. En el norte, las milicias kurdas bloquearon Kirkuk. Y finalmente, el 9 de abril se tomó el control de Bagdad, lo cual se representó en una imagen que dio la vuelta al mundo y sería clave para el inicio de la insurgencia en Irak: el derribo de la estatua de Hussein en la plaza principal de la ciudad. 
Según Chandrasekaran, luego de diez días en Bagdad un miembro de la ORHA, Ronald Adams, regresó a Washington debido a una infección pulmonar. Descubrió que desde el Pentágono, Feith buscaba purgar al partido Baas, establecer a Chalabi como líder y reorganizar al ejército iraquí. Objetivos fatales que Paul Bremer consolidó a través de la dirección de la Autoridad Provisional de la Coalición (CPA, por sus siglas en inglés), a partir del 21 de abril de 2003. Tema a desarrollar en el siguiente apartado.

\section{Autoridad Provisional de la Coalición (APC): error perfecto}

Según el Artículo 43 de la Sección Segunda de la Convención de La Haya de 1899, toda potencia invasora debe cumplir las leyes del país ocupado, salvo cuando sea necesario promover el orden público y la seguridad. De acuerdo a la Resolución 1483 aprobada por el Consejo de Seguridad el 22 de mayo de 2003, la autoridad (EE. UU.) tenía la aprobación para promover "la reconstrucción de la economía y condiciones para un desarrollo sostenible ${ }^{16 "}$.

El trabajo de Garner duró relativamente poco. El 21 de abril de 2003 se instaló la APC con autoridad ejecutiva, legislativa y judicial. $\mathrm{Su}$ director fue Paul Bremer ${ }^{17}$, un veterano del servicio exterior de EE. UU. Este organismo se encargó de promulgar leyes, imprimir papel moneda (billete sin la cara de Hussein), cobrar impuestos, desplegar la Policía, purgar al ejército y gastar los ingresos del petróleo, pues el Programa Petróleo por Alimentos se dio por finalizado y así "sus responsabilidades operacionales, incluyendo todos los proyectos humanitarios establecidos gracias a los ingresos petrolíferos se transfirieron a la APC ${ }^{18}$ ". Un año atrás, Wolfowitz manifestó que el costo de la reconstrucción debería ser mínimo y financiado con los ingresos de la venta del petróleo iraquí (Roberts, 2007, p. 46).

Los planes de Feith fueron ejecutados por Bremer. Según Ismael \& Ismael, fueron dos los principales objetivos de Bremer: aplicar el mercado libre a la economía iraquí y sacar a los miembros del partido Baas de sus cargos militares y oficiales. Estas medidas generaron un colapso en la infraestructura y tejido social iraquí. Aproximadamente 500.000 oficiales fueron despedidos, lo cual elevó la cifra de desempleo entre el $60 \%$ y el $70 \%{ }^{19}$. Asimismo incidió, de manera contundente, en el inicio de la insurgencia, pues muchos de los militares habían luchado en la guerra contra Irán durante la década del ochenta, y habían continuado en las fuerzas de seguridad, porque en Irak su única opción de empleo era la filiación al Partido Baas y a las fuerzas del régimen, y por tanto, su alternativa ante la decisión de la APC fue unirse a la insurgencia (Gause, 2010, p. 157) ${ }^{20}$.

La purga del Partido Baas generó un vacío social y político que, paradójicamente, fue llenado por movimientos pro Baas a nivel tribal, religioso y comunal (Ismael \& Ismael, 2005, p. 617). La respuesta de la APC ante esto fue errónea. A través de un contrato por 236 millones de dólares, una empresa de Carolina del Norte, que poca idea tenía del contexto iraquí, se encargó de los Concejos Municipales.

Los objetivos de la APC no eran acordes con la realidad iraquí. Un país devastado por años de guerra, en donde la imagen de Washington estaba minada entre kurdos y chiitas por su apoyo al régimen suní de Hussein. A los que sumaría también el descontento sunita por la caída del dictador, sobre todo en el occidente del país, en donde creció con fuerza el antiamericanismo y la guerra de guerrillas que se relata más adelante (Gause, 2010, p. 159). Algunos militares tampoco estaban conformes con los planes de la APC. El Mayor General David Paetreus advirtió a Bremer del peligro que implicaba despedir a personal del partido del ejército.

La APC funcionó en la denominada Zona Verde de Bagdad, que era una especie de ciudad americana a escala ${ }^{21}$. Estaba llena de contratistas de Bechtel, General Electric, Halliburton, CSC/ 
Dyncorp, Kellog Brown \& Roth. Los miembros de estas empresas estaban fuertemente protegidos por compañías de seguridad privadas, lo cual hizo aún más difícil convencer a la población civil de que se trataba de una operación de reconstrucción civil (Bjork \& Jones, 2005, p. 787). Algunos diplomáticos con experiencia en el mundo árabe deseaban que existiera una cocina local con trabajadores nativos, pero estos eran una minoría. Gran parte de los trabajadores no había salido de EE. UU., incluso más de la mitad había tenido que sacar el pasaporte para viajar por primera vez a Irak.

¿Cómo se gestionaban los informes? Mark Schroeder fue el analista que los preparaba semanalmente a Bremer. Estaban llenos de gráficos que mostraban un supuesto avance de la APC en las áreas estratégicas que les interesaban: megavatios de electricidad generados, agentes de Policía "locales" entrenados y dinero gastado en labores de reconstrucción. Estos informes iban con copia a Condoleezza Rice y a Donald Rumsfeld. Luego de su estudio, analistas del Pentágono redactaban la información "secreta" y la enviaban a empleados de la APC en Irak. Las gráficas e indicadores eran utilizados en las cifras que ofrecía la Oficina de Relaciones Públicas de la APC, las cuales no coincidían con la realidad; según Chandrasekaran, Schroeder y sus colegas se enteraban de lo ocurrido en Irak a través de Fox News. Es evidente que se trató de construir un discurso (constructivismo) desde el gobierno y los medios, a través de mensajes y supuestos que poco tenían que ver con la realidad en el Irak invadido.

En lo económico la APC también falló: "La más grande liquidación de empresas desde el colapso de la URSS" (Ismael \& Ismael, 2005, p. 617). Se facilitó la entrada a cualquier tipo de producto, mientras que se cerraron las puertas a la producción doméstica del país. Todo esto, a través de unas leyes creadas en septiembre de 2003 que facilitaron la presencia de transnacionales, en una economía cercana al colapso total $^{22}$. Esto fue acompañado de una fuerte ideo- logía de mercado (de nuevo el constructivismo) que hizo énfasis en la privatización (a excepción del sector petrolero) y con reglas laxas para los inversores extranjeros, con lo que se benefició a las compañías estadounidenses como Halliburton pero no a las iraquíes, cayendo en una red de corrupción ante los ojos de la población local (Le Billon, 2005, p. 696). Algo que fue visto como: "la venta comercial del país" (Bjork \& Jones, 2005, p. 785).

Peter MacPherson fue el director de la política económica de la APC. Había dirigido la USAID durante siete años en la era Reagan. Su objetivo era llevar el capitalismo a Irak, crear una Bolsa de Bagdad de primer nivel para comerciar valores y atraer inversión, junto con un fuerte sistema de impuestos. Pero no evidenció que el desempleo rozaba el $40 \%$ o más, y que los iraquíes sentían recelo al pensar en la venta de las empresas nacionales.

Dentro de los reglamentos de la APC, la Orden 37 rebajó los impuestos a las empresas del $40 \%$ al $15 \%$. La Orden 39 permitió a las compañías extranjeras obtener el $100 \%$ de las compañías iraquíes, y podían transferir al exterior todas las ganancias sin la obligación de reinvertir o pagar impuestos. Esta misma orden estableció un plazo de 40 años de arrendamiento para los extranjeros que tomaran empresas o activos del país. La Orden 40 facilitó las condiciones para la banca extranjera en cuanto a inversión y transferencia de valores ${ }^{23}$. Pero se mantuvieron prohibiciones de la era Hussein: la imposibilidad de crear sindicatos o realizar negociaciones colectivas (Ismael \& Ismael, 2005, p. 617).

El desempleo y la política de privatizaciones también elevaron el nivel de violencia. Faez Ghani Aziz, quien era director de la Compañía Estatal de Aceites Vegetales, fue asesinado en la puerta de la fábrica. Días atrás se había negado a contratar a varios desempleados. Al mismo tiempo, Bremer tenía afán en su propósito de iniciar las privatizaciones: "Nuestro objetivo estratégico en los próximos meses será poner 
en marcha una serie de políticas encaminadas a reasignar personas y recursos desde las empresas estatales a empresas privadas más productivas" (Chandrasekaran, 2006a, p. 262).

Tom Holey dirigió la Oficina para el Desarrollo del Sector Privado. Su objetivo era privatizar todas las empresas estatales en un mes. Esta tarea fue imposible debido al elevado riesgo, por lo cual optó por arrendarlas: el modelo aplicado en Kosovo. Pero los problemas de la insurgencia, la falta de electricidad y el aeropuerto de Bagdad cerrado a vuelos internacionales, no permitieron que llegara ningún tipo de socio comercial ${ }^{24}$. Se debe destacar que tanto privatizar como arrendar contradecía la Convención de La Haya de 1899; específicamente el artículo 55, que hace referencia a "las leyes y costumbres de la tierra durante la guerra".

La política económica de la APC se convirtió en una amenaza para todos los sectores de la fragmentada economía iraquí. Esto solo catalizó la feroz resistencia. La educación y cultura corrieron la misma suerte. John Agresto llegó a Bagdad con el propósito de arreglar el sistema universitario. Existían 375.000 estudiantes en 22 campus. $\mathrm{Al}$ igual que otros miembros de la APC, no tenía experiencia en Oriente Medio. Había trabajado con Donald Rumsfeld en la Fundación Nacional para las Humanidades. Dirigía el St. John's College de Santa Fe, colegio en el que la esposa de Rumsfeld había estado en la junta directiva.

Según Agresto, eran necesarios más de dos mil millones de dólares para dar vida a las universidades de Irak. Solo le asignaron ocho millones, provenientes de los fondos de reconstrucción. Había solicitado 130.000 pupitres a USAID pero solo obtuvo 8000: "Soy un neoconservador engañado por la realidad", manifestó, sintió que sus planes habían sido dañados por Washington (Chandrasekaran, 2006a, p. 17). La insurgencia amenazó al personal e impidió las visitas a centros educativos.

La no comprensión de la cultura árabe fue otra gran falla de la APC. Cuando las fuerzas aliadas llegaron a Bagdad, no protegieron los centros culturales, museos y demás monumentos históricos de la rica tradición perteneciente al Califato de Bagdad y la Cultura Asiria, Ismael e Ismael son más radicales al respecto: "El saqueo organizado de bienes culturales, educativos y de salud, y la quema de registros históricos, simbolizó una política deliberada de limpieza cultural" (Ismael \& Ismael, 2005, p. 616). Los soldados solo cuidaron el Ministerio de Petróleo, observaron con indiferencia la destrucción de Bagdad e hicieron caso omiso a los llamados de la población iraquí ${ }^{25}$.

Barbara Bodine, una funcionaria con vasta experiencia diplomática en el Mundo Árabe, sería designada como alcaldesa provisional de Bagdad. Se enteró a través de una fuente personal que material histórico asirio iba a ser robado de las cámaras secretas del Banco Central. De inmediato envió un mensaje de alerta al Departamento de Estado. El funcionario que tomó los mensajes contestó: “¿Qué es el legado asirio?”. Posteriormente, Rumsfeld fue interrogado por los saqueos, a lo que respondió: "La libertad implica desorden" (Chandrasekaran, 2006a, p. 59). El Museo de Bagdad albergaba una de las colecciones más ricas en objetos y manuscritos de civilizaciones antiguas. Se calcula que se perdieron 8500 objetos entre los exhibidos y almacenados, lo cual equivale al $2 \%$ del medio millón total de objetos. "Dada la abrumadora fuerza utilizada y el conocimiento de los antecedentes culturales de Irak por parte de los agresores, es una profunda incompetencia que estas reservas históricas se hayan puesto en riesgo y perdido" (Warren, 2005, pp. 816-817).

El 2 de mayo de 2003 se presentó el hecho más paradójico de la posguerra. G. W. Bush proclamó la "victoria en Irak", a bordo del portaviones Abraham Lincoln con 5000 hombres a bordo. Este hecho fue duramente criticado tanto por la opinión pública estadounidense como por todos los miembros de la ORHA, quienes vieron en la actuación de G. W. Bush una farsa ante el creciente e incontenible caos en Irak. 
No obstante, G. W. Bush no declaró formalmente el fin de la guerra. De acuerdo a la Convención de Ginebra, EE. UU. como potencia ocupante, tenía la obligación de liberar a los prisioneros de guerra y cesar las operaciones contra dirigentes del antiguo régimen. Algo que nunca sucedió, como se vio anteriormente, la persecución contra el partido Baas fue uno de los propósitos de Cheney, Feith, Bremer y Chalabi.

También reconoció que faltaban metas por cumplirse: consolidar la democracia y reconstruir la infraestructura del país (a través de contratos con empresas estadounidenses). En ese momento aún no se había capturado a Hus$\operatorname{sein}^{26}$. Manifestó lo siguiente a la audiencia de EE. UU. y mundial:

Estamos persiguiendo y encontrando líderes del viejo régimen que tendrán que responsabilizarse de sus crímenes. La liberación de Irak es un avance crucial en la campaña contra el terrorismo. Hemos eliminado un aliado de Al Qaeda, y cortado una fuente de financiación del terrorismo. El derrocamiento de Hussein es fundamental para detener la violencia extremista, y el compromiso con la libertad es una tradición para América. Tenemos información de ciertos sitios que deben ser investigados. La transición de una dictadura a una democracia llevará tiempo, pero vale la pena cualquier esfuerzo. Nuestra coalición se quedará hasta que el trabajo esté terminado, y entonces dejaremos un Irak libre. En la batalla de Irak, EE. UU. y sus aliados vencieron, peleamos por la causa de la libertad y la paz del mundo ${ }^{27}$.

El 22 de julio de 2003 la APC y la ONU crearon el Consejo de Gobierno Iraquí (IGC, por sus siglas en inglés). Los miembros fueron escogidos por Paul Bremer, Sergio Vieira de Mello, jefe de la misión de la ONU en el país ${ }^{28}$, y algunos políticos regresados del exilio. También participaron los siete partidos exiliados dominantes (Dodge, 2005, p. 715). Se buscó un equilibrio de poder que representara los clanes del país: trece chiitas, cinco sunitas, un turco y un cristiano (Chalabi estaba en el bloque chiita), y Hamid Majid Mousa fue agregado en representación del Partido Comunista Iraquí, el número posteriormente creció a 25 representantes.

En conclusión, la reconstrucción no tuvo éxito. Nunca se hizo un plan ordenado que involucrara a los iraquíes en los proyectos, los cuales estaban pensados en las empresas contratistas y no en las personas. Como se ha visto en este apartado, los proyectos nunca alcanzaron los estándares esperados, debido a que EE. UU. condicionó la ayuda y se demoró casi un año en entender las fallas en los sectores pobres, hecho que aprovechó Moqtada al-Sadr para reclutar insurgentes en Ciudad Sadr una zona pobre de Bagdad con aproximadamente dos millones de personas que vivían bajo difíciles condiciones (Brown, 2005, p. 768).

En 2004, la reconstrucción pasó del Departamento de Defensa al Departamento de Estado. Las decisiones de la Administración G. W. Bush fueron apresuradas en medio de la imprecisión y fallas de la misión. La toma de Bagdad, el avance sobre algunas ciudades y las capturas de algunos miembros del régimen, no representaron ventaja alguna frente a la "guerra de guerrillas" que se inició en el país y que claramente favoreció a los insurgentes, tal y como se explica a continuación.

\section{La insurgencia: la lucha contra Moqtada al-Sadr y el fracaso en Faluya y Nayaf}

Como se ha visto, varios factores incidieron de forma contundente en la explosión de la insurgencia. Uno de los hechos más impactantes fueron los abusos cometidos en la prisión de Abu Ghraib ${ }^{29}$. El escándalo inició el 13 de enero de 2004, cuando el sargento Joseph Darby 
denunció una serie de abusos a los que fueron sometidos algunos presos. Fueron cometidos por miembros de la Compañía de Policía Militar n. ${ }^{\circ}$ 372, con base en Cumberland, Maryland (temporalmente adjunta a la Brigada de Policía Militar n. ${ }^{\circ} 800$, unidad de reserva con base en Uniondale, Nueva York $)^{30}$ (Murphy, 2004, p. 593).

Esto minó credibilidad y autoridad moral de EE. UU. ante el Mundo Árabe (Baran, 2005) e incluso ante los propios aliados occidentales: "Eventos como Abu Ghraib han brindado elementos al antiamericanismo, una nueva y potente arma en la lucha por los corazones y las mentes" (Walt, 2008). El mismo autor al respecto: "Bush manifestó que Abu Ghraib no refleja la América que conozco (...) el punto no es lo que los americanos piensen sobre la conducta de su nación, es como esa conducta es vista por los otros" (Walt, 2005) ${ }^{31}$. El 13 de mayo de 2004 Kofi Annan manifestó: "No hay duda de que el maltrato a los prisioneros ha causado un daño profundo y real" (Murphy, 2004, p. 596).

El 28 de marzo de 2004 Bremer cometió otro error fatal. Ordenó el cierre del diario $\mathrm{Al}$ Hawzam, el cual era dirigido por el líder chiita Moqtada al-Sadr, hijo del ayatola Muhammad Sadiq al-Sadr, quien fue asesinado, junto con dos de sus hijos, por el régimen de Hussein en 1999. Uno de los argumentos de Bremer para cerrar el periódico fue un reportaje que se publicó en febrero de ese año: "Bremer sigue los pasos de Saddam". Se le acusó de dejar morir de hambre deliberadamente al pueblo iraquí. Dos días antes del cierre, los marines estadounidenses habían matado a 15 iraquíes durante una incursión. Esa misma semana, y luego del cierre del periódico, cuatro contratistas de la firma Blackwater ${ }^{32}$ fueron asesinados en Faluya y sus cuerpos luego fueron colgados en un puente sobre el río Éufrates. También cinco soldados fueron muertos en Habbaniya. Estos hechos fueron ampliamente difundidos por los medios occidentales, generando una fuerte repercusión en la opinión pública estadounidense. Bremer prometió vengar estas muertes.
En abril de 2004 las fuerzas estadounidenses iniciaron la Operación Resolución Vigilante: una fuerte acción en Faluya liderada por el Brigadier General Mark Kimmitt (Gause, 2010, p. 160). Fue un escenario de guerra urbana, irregular, en donde se presentaron más de quinientas muertes civiles. Este hecho provocó una fuerte presión de la opinión pública internacional. Las tropas debieron retirarse y se enfocaron nuevamente en la resistencia chiita en el sur del país.

Ante la compleja situación, Washington y la ONU dieron prioridad a la propuesta de Lakhdar Brahimi. Había cumplido una positiva labor en Afganistán y se le veía como el único mediador capaz de sacar el proceso adelante (Dodge, 2005, p. 717). Su propuesta consistía en crear un gobierno de transición antes de las elecciones de 2005, a partir de la experiencia de cada miembro y no de su filiación política. Una tecnocracia con la habilidad de servir al país y no a los partidos políticos. Su tercer viaje a Bagdad coincidió con el peor momento de violencia que se describe en este apartado: en el norte, la lucha en Faluya y en el sur contra la resistencia de al-Sadr.

La propuesta de Brahimi contaba con el beneplácito del Presidente del Consejo de Seguridad, el alemán Gunter Pleuger, y de Kofi Annan: "Necesitaremos que el Consejo de Seguridad esté con nosotros", manifestó Brahimi. El nuevo Gobierno Provisional tenía la función de gobernar el país hasta las elecciones de enero de 2005. No obstante, Brahimi manifestó: "Bremer es el dictador de Irak: tiene el dinero y la firma, los estadounidenses están gobernando este país".

La presión de la insurgencia y demás errores llevaron a Bremer a querer disolver la APC. El 28 de junio de 2004, la APC transfirió la soberanía al Gobierno Provisional de Irak (Interim Government of Iraq, IGC por sus siglas en inglés). El 28 de junio fue escogido Iyad Allawi como Primer Ministro Interino ${ }^{33}$; Ibrahim al Jaafari, vicepresidente (Dawa); 
Rowsch Shaways, vicepresidente (Partido Democrático Kurdo). Los ministerios fueron al resto de partidos que estaban presentes en el anterior Consejo de Gobierno:

Era la antítesis de lo que planteó Brahimi (...) Parecía que la APC hubiera optado por nombrar a personas claves del Consejo de Gobierno, más que tener el ánimo de garantizar la imparcialidad del proceso electoral (...) En noviembre de 2003 se les consideraba irresponsables e incapaces de dar la mano a la población iraquí, y la APC confió de nuevo en ellos y en sus partidos para la creación del nuevo gobierno (Dodge, 2005, p. 718).

Esta élite demostró una gran incapacidad para cumplir sus objetivos. No logró articular las funciones del gobierno con el antiguo servicio civil, la Policía y el ejército iraquí. Tampoco logró conseguir apoyo en la sociedad ni movilizar a la opinión pública en favor del proceso de reconstrucción nacional. Es evidente que Washington continuó ejerciendo presión para sofocar la insurgencia a través de las fuerzas de seguridad del país con una visión neoconservadora sesgada. Pero el intento resultó infructuoso, a pesar de la legitimidad que le daba el uso de "plenas funciones y autoridad" manifiestas en la Resolución 1546 del Consejo de Seguridad de la ONU del 8 de junio de 2004 (Mattli \& Gasser, 2008, p. 6).

La insurgencia en el "Triángulo sunita y en el sur chiita continuó fuera de control"34. En agosto de 2004 las fuerzas de la coalición decidieron atacar Nayaf. El propósito fue combatir y debilitar al Ejército Mahdi, leal a Moqtda alSadr. La ciudad fue sitiada durante tres meses, pero la victoria nunca llegó, ya que la máxima autoridad chiita en Irak, el Ayatollah al-Sistani, rompió un "débil" acuerdo de cese al fuego, que previamente habían acordado al-Sadr y EE. UU. En una entrevista, miembros de las milicias declararon que la resistencia planeada des- de 2003 no era para luchar contra la invasión, sino contra la ocupación que de seguro se iba a presentar (Ismael \& Ismael, 2005, p. 619). El Comandante del Ejército de EE. UU. Ricardo Sánchez nunca estuvo de acuerdo con la persecución a los chiitas, pero Bremer era quien mandaba (Chandrasekaran, 2006b, p. 40).

Desde ese momento, Al-Sadr se convirtió en uno de los hombres más fuertes en Irak (Nasr, et al., 2007, pp. 41-42). La fuerte resistencia continuó con una alianza entre sunitas y chiitas que debilitó y complicó a las fuerzas extranjeras a través de fuertes ataques en Kufa, Kerbala y Najaf. El 8 de noviembre de 2004 se inició la Operación Furia Fantasma bajo el mando del General Richard Natonsky. Aproximadamente 14.000 soldados atacaron nuevamente a Faluya $^{35}$. Diversas fuentes argumentan que se utilizaron armas prohibidas de forma indiscriminada contra la población civil: fósforo blanco y Mark-77, un agente parecido al Napalm ${ }^{36}$. El 17 de noviembre, la Alta Comisionada de Derechos Humanos de la ONU Louise Arbour pidió una investigación sobre los hechos ocurridos en Faluya, con alta preocupación sobre el uso desproporcionado de la fuerza y el ataque a civiles (Ismael \& Ismael, 2005, p. 624). El fracaso también fue evidente: aunque fueron dados de baja aproximadamente 1500 milicianos, no se pudo capturar a Abu Musab al-Zarqaui ${ }^{37}$, autoproclamado líder de Al Qaeda en Irak; en cambio, algunas fuentes como la Cruz Roja Internacional aseguran que la intervención dejó como saldo 6000 civiles muertos y la ciudad destruida ${ }^{38}$.

Algunos sectores sunitas acusaron al gobierno de Allawi de favorecer a los chiitas. Algunas fuentes indican que pudo participar, junto con los insurgentes de Al-Sadr, en asesinatos de sunitas, y con el apoyo de Irán, país que amplió su esfera de influencia sobre la población chiita de Irak". Al respecto Nasr:

El vacío político que dejó la caída de Hussein permitió que la influencia iraní se extendiera rápidamente 
hacia el sur de Irak. Incrementó el volumen de comercio y el flujo masivo de peregrinos iraníes a las ciudades santas de Irak. Asimismo, se fortalecieron lazos político y de inteligencia. La influencia iraní también se extendió a la burocracia clerical chiita, el aparato de segu-ridad y el sistema político. La guerra (...) allanó el camino para la hegemonía iraní en el Golfo Pérsico (Nasr, et al., 2007, pp. 40-41).

Los políticos iraquíes que regresaron del exilio con la fuerza estadounidense carecían de una imagen favorable frente a sus compatriotas. Eran vistos como simples oportunistas que nunca vivieron las difíciles circunstancias acaecidas durante el régimen de sanciones y bombardeos de la década de los noventa. Algunos reconocieron off the record que nunca imaginaron las dificultades que encontraron a su retorno al país, con una población reacia a ofrecer lealtad política (Dodge, 2005, p. 713).

El 30 de enero de 2005 se realizaron las primeras elecciones pos-Hussein. El ambiente era complejo: ocupación militar, insurgencia e inseguridad (Ismael \& Ismael, 2005, p. 624). El 13 de febrero, la Comisión Electoral Iraquí anunció el resultado del conteo de votos: i) Alianza Unida Iraquí (lista del ayatola Al-Sistani), 51\% y 140 puestos (de 275 posibles); ii) lista Kurda, 26\% y 75 sillas; iii) lista de Allawi, 14\% y 40 puestos, $\mathrm{y}$ iv) otras listas chiitas, 11 puestos (y otros partidos desconocidos tuvieron algunos puestos con poco peso representativo). (Abrahms, 2008)

Se presentaron 7500 candidatos pertenecientes a 117 entidades políticas construidas artificialmente. Eran 117 programas políticos que confundieron a los votantes debido a la inexperiencia electoral, propia de varias décadas de dictadura. De acuerdo a la legislación de la APC, para la elección del presidente, los dos vicepresidentes y el primer ministro eran necesarios dos tercios de la mayoría. Su partido Alianza Unida
Iraquí hizo un acuerdo con los kurdos para obtener la mayoría en la Asamblea Nacional.

Al-Jafari gobernó con poco margen de acción. Trató de reducir a la insurgencia suní, pero fue acusado de sectarismo político por los civiles sunitas e incluso los kurdos. Tampoco fue bien vista su estrecha relación con Irán y con Moqtda al-Sadr. Y a pesar de que el 12 de febrero de 2006 ganó la consulta interna de su partido frente a Adil Abdul-Mahdi, se hizo imposible su reelección, por lo cual se vio obligado a renunciar. El 22 de abril de ese año fue elegido como Primer Ministro Nuri al-Maliki, una figura menos polémica que contaba con el visto bueno de sunitas, kurdos y la pequeña clase industrial. El 7 de marzo de 2010 se presentó para ser reelegido. Luego de un largo proceso lleno de dudas, impugnaciones y oscuras alianzas, el 7 de noviembre de ese año se consolidó un acuerdo entre los principales partidos políticos iraquíes para reelegir como Primer Ministro a Nuri al-Maliki y como presidente a Yalal Talabani.

Tres meses antes, el 31 de agosto, al-Maliki se dirigió al pueblo iraquí manifestando que celebraba la retirada de la tropa estadounidense: "Irak es hoy soberano e independiente". No obstante, una buena conclusión de este apartado está en palabras de Scheuer:

Olvidamos que ninguno de los vecinos de Irak comparte nuestro objetivo de ver un país democrático y secular, y que el fin de Saddam llevaría de forma inevitable a una lucha sectaria entre chiitas y sunitas, en Irak, Irán y el resto de Estados de la región, y todos ellos contra nosotros. Y fallamos en cerrar las fronteras de Irak (Scheuer, 2007, p. 245).

\section{Conclusiones}

El esfuerzo de G. H. Bush y su equipo en Irak fue representativo de una corriente de pensamiento arraigada en Washington. Los hechos 
de la intervención y la compleja posguerra demostraron que la "venta" de un modelo democrático en Oriente Medio fracasó.

Las mentiras de Powell ante el Consejo de Seguridad, el poco margen de maniobra de Garner y la incapacidad de Bremer prepararon el espacio político para un grupo de exiliados con poca credibilidad. Irán, Al Qaeda y el fundamentalismo radical encontraron espacio libre en una zona que Hussein había blindado desde la Guerra Fría.

Desde entonces, el tema iraquí ha afectado a todos los sectores políticos en Washington. El costo económico y en vidas, junto a la lucha energética de la zona en donde juegan otras potencias, se convirtió en uno de los grandes desafíos para la política exterior de EE. UU. en el siglo XXI.

Paradójicamente, la superioridad de recursos en términos realistas no le permitió a EE. UU. triunfar en Irak. El unilateralismo aplicado durante la Administración de G. W. Bush hizo daño a la estructura de gobernanza global creada desde el fin de la Segunda Guerra Mundial, y los discursos del éxito en Irak rápidamente fueron desvirtuados por los hechos, que como se ha visto, evidenciaban una realidad diferente, alejada del "sueño democrático" de Irak y Oriente Medio en el siglo XXI.

\section{Notas}

${ }^{1}$ Sobre la evolución de Al Qaeda, véase el sólido trabajo de Wright (2006): The Looming Tower. Al-Qaeda and the Road to 9/11.

${ }^{2}$ Antes del inicio de las operaciones de EE. UU. en Afganistán, algunos intelectuales estadounidenses como Noam Chomsky fueron escépticos sobre la presunta relación entre $\mathrm{Al}$ Qaeda y los atentados del 11-S. Durante una entrevista realizada una semana después de los ataques, por la cadena ABC News, Chomsky manifestó lo siguiente en Boston: "No tenemos evidencia fidedigna sobre la participación directa de Bin Laden, y esto incluye, supongo, a los servicios de inteligencia de EE. UU.".

${ }^{3}$ El 8 y 9 de marzo de 2001, el Mullah Mohammed Omar, máxima autoridad del Régimen Talibán ordenó la destrucción de las estatuas. El enviado especial de la UNESCO a Afganistán Pierre Lafrance le confirmó la noticia al Director General Koichiro Matsuura (1999-2009). Las estatuas tenían una antigüedad de 1500 años. El argumento de los talibanes era abolir la "idolatría". Incluso una delegación de la Conferencia Islámica, organización que no reconocía al Régimen Talibán, trató de impedir el criticado hecho, al igual que lo hizo Irán.

${ }^{4}$ Por su ubicación geográfica, Afganistán ha sido un buffer-state por antonomasia. Actualmente, es vital para los intereses de Washington en la región debido a su cercanía con Pakistán, India, China y Rusia, actores que desempeñan un papel preponderante en la configuración del equilibrio de poder en el sistema internacional.

${ }^{5} \mathrm{Al}$ respecto, véase el interesante trabajo: Anti-Americanisms in World Politics (Katzenstein \& Keohane, 2007).

${ }^{6}$ En mayo de 2002, el Subsecretario de Estado John Bolton manifestó que otros Estados deberían hacer parte de dicho eje: Libia, Siria y Cuba, y agregó: "América está determinada a prevenir una nueva ola de terror". Recuperado el 4 de febrero de 2010 de http://news. bbc.co.uk/2/hi/americas/1971852.stm

${ }^{7}$ No obstante, la fecha de orden de ataque a Irak es compleja de definir. Gause cita el trabajo de Woodward Bush at War, en el que manifiesta que G. W. Bush el 12 de septiembre de 2001, ordenó al Oficial Contraterrorismo Richard Clarke que buscara vínculos entre los ataques y el Irak de Hussein. Asimismo, el 17 de septiembre manifestó ante el National Security Council que creía que Irak estaba implicado, pero que no lo 
atacaría de momento porque no tenía evidencia. Este día, Bush ordenó la elaboración de una estrategia de guerra contra Afganistán, la cual incluía el inicio de posibles opciones para invadir a Irak (Gordon \& Trainor, 2006, p. 17, citados en Gause, 2010, p. 192). No obstante, Woodward establece el 21 de noviembre de 2001 como el inicio del plan de guerra hacia Irak (Woodward, 2002, pp. 1-3, 30, citado en Gause, 2010, p. 191). El 28 de diciembre se discutió con el General Tommy Franks la posibilidad de invadir a Irak.

${ }^{8}$ Luego de un proceso electoral complejo, G. H. Bush se posesionó como presidente de EE. UU. con una débil imagen política. El exejecutivo de la empresa familiar Arbusto-Bush Exploration consolidó un staff neoconservador compuesto por algunos personajes que habían desempeñado cargos importantes durante la Administración de su padre, G. H. Bush (19891993). El equipo original de neocons estaba compuesto por: Donald Rumsfeld, Secretario de Defensa; Douglas Feith, Subsecretario de Defensa; Paul Wolfowitz, Diputado del Secretario de Defensa; Elliot Abrams, Asistente Especial del Presidente y del Consejo de Seguridad Nacional; Paula Dobriansky, Subsecretaria de Estado para Asuntos Internacionales; Richard Armitage, (US) Diputado del Secretario de Estado; Robert Zoellick, (US) Representante de Comercio; John Bolton, (US) Subsecretario de Estado para el Control de Armas y Seguridad Internacional; David Wurmser, ayudante especial del Subsecretario Bolton, y Richard Perle, presidente del Comité de Defensa del Pentágono. Otros tenían fuertes nexos con el sector petrolero, como por ejemplo el Vicepresidente Dick Cheney (Harken) (Khalidi, 2004, p. 66).

${ }^{9}$ Las segundas reservas más grandes de petróleo en el mundo, calculadas en 110 billones de barriles, el 12\% de la oferta mundial.

${ }^{10}$ Extracto citado por Dunn (2003, 29 de enero). De The State of the Union Addres, The New York Times.
${ }^{11}$ De esta organización también hace parte Sharif Ali bin Al Hussein, líder del Movimiento por la Monarquía Constitucional, quien ha pretendido recobrar la figura real en Irak, argumentando que de esta manera se podría unificar más fácilmente al país. Es primo de Faisal II, quien fue derrocado y asesinado por una turba en Bagdad en 1958. En entrevista en el diario El País, manifestó que Hussein, quien ya tenía armas químicas y biológicas, estaba dispuesto a hacer lo necesario para desarrollar armas nucleares. Asimismo, subrayó que no era necesaria una intervención extranjera en Irak. (2010, 5 de julio). Saddam Hussein es el hombre más peligroso del mundo. Diario El País. Recuperado de http://www.elpais.com/articulo/reportajes-/ Sadam/Husein/hombre/peligroso/mundo/elpep usocdmg/20021110elpdmgrep_4/Tes. No obstante, algunos manifiestan, de acuerdo con estudios genealógicos, que el verdadero aspirante al trono en la Dinastía Hachemí es el Príncipe Zeid Ra'ad Zeid Al-Hussein, nacido en Jordania y quien ha tenido una destacada vida como diplomático en la ONU y en la Corte Penal Internacional (CPI).

${ }^{12}$ (2009, 9 de septiembre). EE. UU. siempre ha visto en el líder del Congreso Nacional Iraquí a un demócrata. Diario El País. Recuperado de http://www.elpais.com/articulo/internacional/Chalabi/eterno-/enemigo/Sadam/ elpepiint/20030414elpepiint_12/Tes

${ }^{13}$ A pesar de que existe un sentido de identidad entre ciudadanos iraquíes, es un país fuertemente dividido entre las etnias predominantes: chiitas, sunitas y kurdos: "La división social entre estos grupos fue exacerbada por las políticas del régimen de Hussein, quien la utilizó para mantenerse en el poder" (Bellin, 2004, p. 598).

${ }^{14}$ En tono sarcástico, la ORHA era llamada Organization of Really Hopeless Americans.

15 Autores como Dodge son escépticos sobre los planes de Garner, pues: "Ninguno de los escenarios planeados ocurrieron" (Dodge, 2005, p. 712). 
${ }^{16}$ Sitio web del Consejo de Seguridad de la ONU (2009, 4 de septiembre). Recuperado de http:// daccess-ods.un.org/TMP/ 4436946.51126862.html

${ }^{17}$ El diplomático posteriormente publicó un libro en el que relata su gestión en Irak: $M y$ Year in Iraq. The Struggle to Build a Future of Hope (Bremer \& McConnell, 2006).

${ }^{18}$ Sitio web de la página principal de las operaciones de mantenimiento de la paz (2009, 5 de diciembre). Recuperado de http://www. un.org/spanish/Depts/dpko/yir03/Iraq.htm

${ }^{19}$ Gause calcula en 300.000 los soldados que se dedicaron a la insurgencia (Gause, 2010, p. 159).

${ }^{20}$ Entre los miembros del Baas se encontraban asesores de los ministerios, 15.000 profesores y viejos soldados del partido que eran miembros honorarios, luego de pasar años como prisioneros de guerra en campos de detención iraníes (Chandrasekaran, 2006b, p. 37).

${ }^{21}$ La emisora que operaba se denominó Freedom Radio, en dial 107.7 FM. Su programación incluyó clásicos del rock y mensajes de aliento para el personal. Una clara estrategia de soft power.

${ }^{22} \mathrm{El}$ análisis de Bellin justifica el accionar de la CPA. Manifiesta que el Estado iraquí bajo el mandato de Hussein se basaba en corrupción y en lealtades políticas. Por tanto, instituciones claves como la Policía y el sistema judicial deberían haber sido corregidas desde la base. Asimismo, considera que las instituciones políticas no tienen que ser nativas para ser aceptables, dando como ejemplo las imposiciones institucionales a Japón y Alemania luego de ser derrotados tras la Segunda Guerra Mundial (Bellin, 2004, pp. 599-606).

${ }^{23}$ El sitio web de la Autoridad Provisional de la Coalición se mantiene activo en la red. En este se encuentran las órdenes y reglamentos expedidos durante su período de manejo del país. En la página de inicio se aclara que no se actualiza y que permanece, solamente, con propósito de archivo histórico. (2010, 6 de diciembre). Recuperado de http://www.iraqcoalition.org/

${ }^{24}$ Posteriormente, Holey fue enviado con el ex secretario de Estado James Baker a buscar que diferentes países condonaran la deuda externa iraquí.

${ }^{25}$ Tim Carney y Robin Raphael, quien iba a ser el Ministro de Comercio, observaron el saqueo en las calles de Bagdad, desde Kuwait. Cuando el equipo de la ORHA aún no se había trasladado a Irak.

${ }^{26}$ Hussein fue capturado el 14 de diciembre de 2003 en Tikrit. Sus hijos Uday y Qusay murieron el 31 de julio de 2003 en Mosul, luego de un bombardeo de la fuerza aliada sobre su escondite. Ese mismo año, el 25 de abril, fue capturado el viceprimer ministro y canciller del régimen Tarek Aziz. El 26 de abril capturaron al ex director de Operaciones de Servicios Secretos del régimen Faruk Higazi, en la frontera con Siria. El 27 de abril fue arrestado Mahamed Al Zubaidi, exiliado iraquí que se había autoproclamado Alcalde Bagdad y quien daba órdenes a empleados municipales. El 30 de abril se rindió Walid Hamid Taufik, exgobernador de Basora y n. ${ }^{\circ} 44$, en la lista de 55 fugitivos de EE. UU.

${ }^{27}$ Más información al respecto en el diario El País (2011, 1 de enero). "Bush proclama que, tras la victoria en Irak, la guerra contra el terrorismo continúa". Recuperado de http:// www.elpais.com/articulo/internacional/-Bush/ proclama/victoria/Irak/guerra/terrorismo/continua/elpepiopi/20030503elpepiint_5/Tes

${ }^{28}$ El 19 de agosto de 2003 hizo explosión una bomba al frente del Hotel Canal, en donde se encontraba la sede de la ONU. Fallecieron 21 personas y quedaron heridas 100. Entre las víctimas estaban Sergio Vieira de Mello y 
la portavoz Nadia Younes. Este hecho conmocionó a la opinión pública mundial, ya que para muchos Vieira era visto como el posible sucesor de Annan en la Secretaría General de la ONU. El 6 de junio de 2009 fue detenido Ali Hussein al-Azzawi, ex piloto de la empresa estatal Iraqi Airways. Confesó haber orquestado el atentado, además era el encargado de articular las redes de Al Qaeda en Irak y en algunas zonas de Europa.

${ }^{29}$ El General Ricardo Sánchez ordenó que el Comando Central seleccionara un oficial para que investigara los hechos en Abu Ghraib. El 31 de enero de 2004 esta misión fue asignada al Mayor General Antonio Taguba, quien lideró un equipo que revisó fotografías, videos y entrevistó aproximadamente a cincuenta testigos. Su informe, conocido como El Reporte Taguba, corroboró los malos tratos, torturas y vejaciones. Entre 2003 y 2004 el ejército de EE. UU. abrió más de noventa investigaciones sobre abusos a detenidos y civiles en Irak y Afganistán por parte de soldados estadounidenses (Murphy, 2004, p. 595).

${ }^{30}$ Agentes de la CIA y contratistas de empresas de seguridad solicitaron a los guardias condiciones físicas y mentales favorables para los interrogatorios (Murphy, 2004, p. 595).

${ }^{31}$ En abril se presentaron informes especiales en el programa 60 minutos, de la Cadena CBS. Asimismo, en The New Yorker el reconocido periodista Seymur Hersh escribió un artículo al respecto haciendo énfasis en las diversas torturas perpetradas allí. El escándalo político afectó a Rumsfeld y al Partido Republicano. Varios militares implicados como Charles Grane y Lynndie England fueron sentenciados y enviados a prisión. En mayo de 2005, la Brigadier General Janis Karpinski perdió su rango y pasó a coronel. Posteriormente, manifestó que las órdenes venían de arriba y que estaba dispuesta a acusar al propio Rumsfeld sobre los hechos. Esta situación es similar al debate suscitado por los abusos cometidos contra prisioneros en la cárcel de Guantánamo en Cuba (la mayoría capturados en Afganistán luego de 2001) a quienes se les negó el debido proceso, entro otros abusos más (Walt, 2005).

${ }^{32}$ Otras empresas de seguridad privadas contratadas por la APC fueron: DynCorp International, ArmorGroup International y Custer Battles. De acuerdo a la Orden n. ${ }^{\circ} 17$ de la $\mathrm{CPA}$, no estaban sometidas a la ley iraquí. Existen pruebas de que utilizaron armas prohibidas, diferentes de la munición estándar: "Thomas esperaba disparar de nuevo, pero su blanco se había desplomado (...) su cadera estaba destrozada y su abdomen, pulverizado por el lugar donde había salido la bala (...) en lugar de un núcleo de plomo, estos proyectiles estaban hechos con una mezcla de varios metales, incluido el platino, de modo que podían atravesar una coraza de acero y en cambio se hacían añicos en la carne, causando unas heridas catastróficas" (Chandrasekaran, 2006a, p. 176). El uso de este tipo de munición, con punta blanda sin cartucho metálico, genera graves daños cuando entra en la carne, su uso está prohibido por diversos tratados internacionales. El 27 de octubre de 2005 se publicó un video en el que contratistas de la empresa Aegis disparaban a conductores iraquíes, riéndose y escuchando canciones de Elvis Presley.

${ }^{33}$ Ocupó este cargo hasta que se realizaron las primeras elecciones, posteriores a la intervención, el 30 de enero de 2005. El partido de Allawi perdió, pese al apoyo de Washington, y el 7 de abril entregó el poder a Ibrahim alJaafari, quien gobernó hasta el 20 de mayo de 2006, cuando entregó el poder a Nuri al-Maliki, quien ocupa el cargo desde entonces, en medio de un panorama político complejo.

${ }^{34}$ Esta zona se encuentra al noroeste de Bagdad. Es altamente poblada y la mayoría de la población es sunita. Las principales ciudades son Ramadi, Tikrit, Baquba, Mosul y Faluya.

${ }^{35}$ El documental Fallujah, The Hidden Massacre, dirigido por Sigfrido Ranucci y Mau- 
rizio Torrealta, presentado el 8 de noviembre de 2005 en la RAI, asegura que se usaron armas químicas durante el ataque a Faluya en 2004. Para ello cuenta con los testimonios de dos ex militares que estuvieron en la operación: Jeff Englehart y Garret Reppenhagen. Video disponible en línea. Recuperado el 9 de noviembre de 2010, de http://www.rainews24.rai.it/ran24/ inchiesta/video/fallujah_ING.wmv

36 "Fuerzas de EE.UU. 'usaron armas químicas' durante el asalto a la ciudad de Faluya". Página web del diario The Independent (2010, 4 de noviembre). Recuperado de http://www.independent.co.uk/news-/world/-middle-east/-usforces-used-chemical-weapons-during-assaulton-city-of-fallujah514433.html

\section{Referencias}

Abrahms, M. (2008). What Terrorists Really Want: Terrorist Motives and Counterterrorism Strategy. International Security, 32(4), pp. 78-105.

Apple, R. W. (2001, 09 - 14). The New York Times.

Baran, Z. (2005). Fighting the War of Ideas. Foreign Affairs, 84(6), pp. 68-78.

Bellin, E. (2004). The Iraqi Intervention and Democracy in Comparative Historical Perspective. Political Science Quarterly, 119(4), pp. 595-608.

Bjork, K., \& Jones, R. (2005). Overcoming Dilemmas Created by the 21 st Century Mercenaries: conceptualising the use of private security companies in Iraq. Third World Quarterly, 26, pp. 777-796.

Bremer, L. P., \& McConnell, M. (2006). My Year in Iraq. The Struggle to Build a Future of Hope. New York: Simon \& Schuster.

Brown, R. (2005). Reconstruction of Infrastructure in Iraq: end to a means or means to an end? Third World Quarterly, 26, pp. 759-775.

Chandrasekaran, R. (2006a). Vida imperial en la Ciudad Esmeralda. Barcelona: RBA Libros.

Chandrasekaran, R. (2006b). Who Killed Iraq? Foreign Policy, pp. 36-43.

Dodge, T. (2005). Iraqi Transitions: from regime change to state collapse. Third World Quarterly, 26, pp. 705-721.

Dunn, D. H. (2003). Myths, Motivations and 'Misunderestimations': the Bush Administration and Iraq. International Affairs, 79(2), pp. 279-297.

Fuentes, C. (2003, abril/junio). ¿Qué tal? "Voces contra la guerra". Revista Nueva Gaceta, 6 .

Gause, F. G. (2010). The International Relations of the Persian Gulf. New York: Cambridge University Press.

Gordon, M. R., \& Trainor, B. E. (2006). Cobra II: The inside story of the invasion and occupation of Iraq. New York: Random House.

Hirsh, M. (2002). Bush and the World. Foreign Affairs, 81(5), pp. 18-43.

Ismael, T., \& Ismael, J. (2005). Whither Iraq? Beyond Saddam, sanctions and occupation. Third World Quarterly, 26, pp. 609-629.

Katzenstein, P., \& Keohane, R. O. (2007). AntiAmericanisms in World Politics. Ithaca New York: Cornell University Press.

Keohane, R. O. (2002). The Globalization of Informal Violence, Theories of World Politics and the "Liberalism of Fear". Dialog-IO, pp. 29-43. 
Kupchan, C. A. (2003). The End of the American Era: A. Knopf.

Le Billon, P. (2005). Corruption, Reconstruction and Oil Governance in Iraq. Third World Quarterly, 26, pp. 685-703.

Mattli, K., \& Gasser, J. (2008). Acción neutral, imparcial e independiente: clave para la aceptación del CICR en Irak. International Review of the Red Cross, 869.

Murphy, S. D. (2004). US Abuse of Iraqi Detainees at Abu Ghraib Prison. American Journal of International Law, 98, pp. 591-596.

Nasr, V.; Filkins, D.; Byman, D.; Frum, D.; Tsang, S.; Ottaway, M., et al. (2007). Who Wins in Iraq? Foreign Policy(159), pp. 38-51.

Roberts, A. (2007). The War We Deserve. Foreign Policy, 163, pp. 45-50.

Rumsfeld, D. H. (2002). Transforming the Military. Foreign Affairs, 81(3), pp. 20-32.
Scheuer, M. (2007). Imperial hubris: why the West is loosing the war on terror. Dulles, Virginia: Potomac Books.

Sohr, R. (2002). Las Guerras que nos esperan: EE. UU. Ataca.: Ediciones B.

Walt, S. M. (2005). Taming American Power. Foreign Affairs, 84(5), pp. 105-120.

Walt, S. M. (2008, March 18). Five Years and Counting: Ten Unpleasant Truths about the War in Iraq.

Warren, J. (2005). War and the Cultural Heritage of Iraq: a sadly mismanaged affair. Third World Quarterly, 26, pp. 815-830.

Wills, G. (2010). Bomb Power: The Modern Presidency and the National Security State. New York: Penguin Press.

Woodward, B. (2002). Bush At War. New York: Simon and Schuster.

Wright, L. (2006). The Looming Tower. Al Qae$d a$ and the Road to 9/11. New York: Vintage Books. Random House. 